\title{
New Singular and Nonsingular Colliding Wave Solutions in Einstein-Maxwell-Scalar Theory.
}

\author{
Ozay Gurtug, Mustafa Halilsoy and Izzet Sakalli \\ Department of Physics, Eastern Mediterranean University \\ G. Magusa, North Cyprus, Mersin 10 - Turkey \\ email: ozay.gurtug@emu.edu.tr
}

\begin{abstract}
A technique is given to generate coupled scalar field solutions in colliding Einstein-Maxwell (EM) waves. By employing the Bell - Szekeres solution as seed and depending on the chosen scalar field, it is possible to construct nonsingular solutions. If the original EM solution is already singular, addition of scalar fields does not make the physics any better. In particular, scalar field solution that is transformable to spherical symmetry is plagued with singularities.
\end{abstract}

\section{INTRODUCTION}

Colliding plane waves (CPWs) in general relativity is known to result either in an all encompassing spacelike singularity or an extendible Cauchy horizon $(\mathrm{CH})[1-3]$. (To be more precise, this classification must be suplemented by the rare class of null singularities). Within this context colliding Einstein-Maxwell (CEM) waves is studied in greater detail $[1,4,5,6]$. While the collision of scalar waves is also known to a certain extend $[7,8]$, the collision of Einstein -Maxwell-Scalar (CEMS) waves has not been investigated in detail. Even dilaton and axion have attracted interest enough to necessiate in retrospect study of scalar fields in its own right. By scalar field it is implied a massless scalar field with minimal coupling, which is the simplest type among existing fields to couple gravity and electromagnetic (em) fields. In recent decade the effect of such a massless scalar field, either in collapse under its own gravity or under the effect of a black hole both have been among popular topics in general relativity $[9,10]$. Minimal coupling to gravity makes a scalar field an indespensable test field for the perturbation studies. To test the stability of a $\mathrm{CH}$ formed in CEM waves we construct CEMS solutions and check whether $\mathrm{CH}$ remains as $\mathrm{CH}$ or transforms into a spacetime singularity. This involves the back reaction effect of the scalar field and must be considered as a stronger case compared to any perturbation analysis. Being inspired by the richness of the CPW spacetimes some researchers investigated mini black hole formation in a laboratory by colliding highly energetic particles [11] that can be considered analogues model of CPWs. All these aspects motivated us to inspect the scalar field effects when coupled in collision with gravity and em fields.

In this paper we consider first the CEM solution of Bell - Szekeres (BS) [4] as seed and construct by the $M-$ shift method, scalar field solutions coupled with it. As a sec- 
ond example we consider the solution found by Hogan, Barrabes and Bressange (HBB) [12]. This solution represents an example of colliding impulsive gravitational wave with a wavepacket consisting of superposed impulsive gravitational wave with an em shock wave. Since this is already a singular solution the added scalar field serves only to modify the colliding wavepackets. Our final example is a scalar field solution that is transformable to the spherically symmetric Penney solution [13].

The paper is organized as follows. In section II we introduce and prove the $M-$ shift method for generating scalar field solutions. In sections III and IV we apply our method to the BS and HBB metrics, respectively. Section V contains a singular solution from the outset because it has been considered isometric to the spherically symmetric geometry. We conclude the paper with discussion in section VI.

\section{A METHOD FOR GENERATING CEMS SOLUTIONS FROM ANY KNOWN CEM SOLUTION.}

In this section we give a simple proof that when given any solution in CEM theory we can generate a class of CEMS solutions with prescribed properties. A similar technique had been used long time ago in the Weyl-Papapetrou form of stationary axially symmetric gravitational fields [14]. The similarity between this form of the metric and the metric of CPWs serves to extend the technique automatically to the latter.

The metric of CPWs in general is given by

$$
d s^{2}=2 e^{-M} d u d v-e^{-U}\left[\left(e^{V} d x^{2}+e^{-V} d y^{2}\right) \cosh W-2 \sinh W d x d y\right]
$$

in which it is understood that all metric functions are at most functions of the null coordinates $u$ and $v$. We also add that whatever we prove for the EM system a reduced version is also valid for the vacuum CPWs. By choosing an em potential one form

$$
\tilde{A}=\tilde{A}_{\mu} d x^{\mu}=A d x+B d y
$$

where $A$ and $B$ are potential functions to be determined and a scalar field $\phi$, the CEMS system can be generated from the Lagrangian density

$$
\begin{gathered}
L=e^{-U}\left(M_{u} U_{v}+M_{v} U_{u}+U_{u} U_{v}-W_{u} W_{v}-V_{u} V_{v} \cosh ^{2} W-4 \phi_{u} \phi_{v}\right) \\
-2 k\left[\left(B_{u} B_{v} e^{V}+A_{u} A_{v} e^{-V}\right) \cosh W\right. \\
\left.+\left(A_{u} B_{v}+A_{v} B_{u}\right) \sinh W\right]
\end{gathered}
$$

The constant $k$ denotes a coupling constant which can be fixed as unity. Variational principle of the action $S$ defined by (and suppressing the $x, y$ coordinates)

$$
S=\int L d u d v
$$

yields the following CEMS equations 


$$
\begin{aligned}
U_{u v}= & U_{u} U_{v} \\
2 M_{u v}= & -U_{u} U_{v}+W_{u} W_{v}+V_{u} V_{v} \cosh ^{2} W+4 \phi_{u} \phi_{v} \\
2 V_{u v}= & U_{v} V_{u}+U_{u} V_{v}-2\left(V_{u} W_{v}+V_{v} W_{u}\right) \tanh W \\
& -2 k \operatorname{sech} W\left(\bar{\Phi}_{0} \Phi_{2}+\bar{\Phi}_{2} \Phi_{0}\right) \\
2 W_{u v}= & U_{v} W_{u}+U_{u} W_{v}+2 V_{u} V_{v} \cosh W \sinh W \\
& +2 k i\left(\bar{\Phi}_{0} \Phi_{2}-\bar{\Phi}_{2} \Phi_{0}\right) \\
2 \phi_{u v}= & U_{v} \phi_{u}+U_{u} \phi_{v} \\
2 A_{u v}= & V_{v} A_{u}+V_{u} A_{v}-\tanh W\left(W_{v} A_{u}+W_{u} A_{v}\right) \\
& -e^{V}\left[2 B_{u v} \tanh W+W_{u} B_{v}+W_{v} B_{u}\right] \\
2 B_{u v}= & -V_{v} B_{u}-V_{u} B_{v}-\tanh W\left(W_{v} B_{u}+W_{u} B_{v}\right) \\
& -e^{V}\left[2 A_{u v} \tanh W+W_{u} A_{v}+W_{v} A_{u}\right]
\end{aligned}
$$

Here $\Phi_{0}$ and $\Phi_{2}$ are the Newman-Penrose spinors for em fields defined by

$$
\begin{aligned}
\Phi_{2}= & \frac{e^{U / 2}}{\sqrt{2}}\left[e^{-V / 2}\left(i \sinh \frac{W}{2}-\cosh \frac{W}{2}\right) A_{u}\right. \\
& \left.+e^{V / 2}\left(i \cosh \frac{W}{2}-\sinh \frac{W}{2}\right) B_{u}\right] \\
\Phi_{0}= & \frac{e^{U / 2}}{\sqrt{2}}\left[e^{-V / 2}\left(i \sinh \frac{W}{2}+\cosh \frac{W}{2}\right) A_{v}\right. \\
& \left.+e^{V / 2}\left(i \cosh \frac{W}{2}+\sinh \frac{W}{2}\right) B_{v}\right]
\end{aligned}
$$

The remaining equations corresponding to $R_{u u}=-T_{u u}$ and $R_{v v}=-T_{v v}$ which do not follow from the variational principle, namely

$$
\begin{aligned}
2 U_{u u}-U_{u}^{2}+2 M_{u} U_{u} & =W_{u}^{2}+V_{u}^{2} \cosh ^{2} W+4 \phi_{u}^{2}+4 k \Phi_{2} \bar{\Phi}_{2} \\
2 U_{v v}-U_{v}^{2}+2 M_{v} U_{v} & =W_{v}^{2}+V_{v}^{2} \cosh ^{2} W+4 \phi_{v}^{2}+4 k \Phi_{0} \bar{\Phi}_{0}
\end{aligned}
$$

are automatically satisfied by virtue of the other equations. Thus the foregoing sets of equations (5 -13) give the complete set of CEMS equations. The equation (13) actually are the guiding equations for us to state the following

Theorem 1 Given an EM metric satisfying the above equations (5-13) with zero scalar field $(\phi=0)$, we can generate solutions with $\phi \neq 0$ by making a shift in the metric function $M$ (i.e. the $M$ - shift) in accordance with

$$
M \longrightarrow \widetilde{M}=M+\Gamma
$$

where the shift function $\Gamma$ must satisfy

$$
\begin{aligned}
\Gamma_{u} U_{u} & =2 \phi_{u}^{2} \\
\Gamma_{v} U_{v} & =2 \phi_{v}^{2}
\end{aligned}
$$


Proof: We observe easily from the pair of eqs.(13) that substitution of $M \longrightarrow \widetilde{M}$, and by virtue of (15) cancels the scalar field from both sides. Further, the integrability of (15), i.e. $\Gamma_{u v}=\Gamma_{v u}$ imposes the scalar field equation

$$
2 \phi_{u v}-U_{v} \phi_{u}-U_{u} \phi_{v}=0
$$

as a constraint condition. This leaves no trace of the scalar field which completes the proof. We can justify the $M-$ shift also by employing the action principle which turns out to yield total divergences. In conclusion, if we have a CEM solution consisting of $(U, M, V, W, A, B)$ then we obtain a CEMS solution expressed by $(U, M+\Gamma, V, W, A, B, \phi)$ where $\Gamma$ is obtained as a line integral

$$
\Gamma=2 \int \frac{\phi_{u}^{2}}{U_{u}} d u+2 \int \frac{\phi_{v}^{2}}{U_{v}} d v
$$

In this technique em potentials and the metric functions $U, V$ and $W$ remain unchanged. Also in practice, the line integral (17) is (except in very particular cases) of little use. Transforming from the null coordinates $(u, v)$ to new types of coordinates $(\tau, \sigma)$, however, we get more advantage toward solutions in closed form. One such useful set of coordinates is defined by

$$
\begin{aligned}
\tau & =u \sqrt{1-v^{2}}+v \sqrt{1-u^{2}} \\
\sigma & =u \sqrt{1-v^{2}}-v \sqrt{1-u^{2}}
\end{aligned}
$$

which transforms the relavant part of the metric as

$$
\frac{d \tau^{2}}{\Delta}-\frac{d \sigma^{2}}{\delta}=\frac{4 d u d v}{\sqrt{1-u^{2}} \sqrt{1-v^{2}}}
$$

with

$$
\begin{aligned}
\Delta & =1-\tau^{2} \\
\delta & =1-\sigma^{2}
\end{aligned}
$$

In terms of these new coordinates the scalar field equation (16) takes the form

$$
\left(\Delta \phi_{\tau}\right)_{\tau}-\left(\delta \phi_{\sigma}\right)_{\sigma}=0
$$

while the $\Gamma$ eq.s (15) become

$$
\begin{aligned}
& \left(\tau^{2}-\sigma^{2}\right) \Gamma_{\tau}=2 \Delta \delta\left(\tau \phi_{\tau}^{2}+\frac{\tau \delta}{\Delta} \phi_{\sigma}^{2}-2 \sigma \phi_{\tau} \phi_{\sigma}\right) \\
& \left(\sigma^{2}-\tau^{2}\right) \Gamma_{\sigma}=2 \Delta \delta\left(\sigma \phi_{\sigma}^{2}+\frac{\sigma \Delta}{\delta} \phi_{\tau}^{2}-2 \tau \phi_{\tau} \phi_{\sigma}\right)
\end{aligned}
$$

The advantage we have obtained by this change of coordinates is that the scalar field equation (21) admits an infinite class of seperable solutions which were not so obvious in the original null coordinates. 
A general class of separable solution for the scalar field $\phi$ is given by [1]

$$
\phi(\tau, \sigma)=\sum_{n}\left\{a_{n} P_{n}(\tau) P_{n}(\sigma)+b_{n} Q_{n}(\tau) Q_{n}(\sigma)+c_{n} P_{n}(\tau) Q_{n}(\sigma)+d_{n} P_{n}(\sigma) Q_{n}(\tau)\right\}
$$

Where $P$ and $Q$ are the Legendre functions of the first and second kind, respectively and

$a_{n}, b_{n}, c_{n}$ and $d_{n}$ are arbitrary constants. Although this expression for $\phi(\tau, \sigma)$ together with the integrals (22) solve the scalar field problem mathematically, we must impose also some physical conditions. The Cauchy data to be imposed as physical input must be acceptable. This discards from the outset any diverging solutions for the scalar field or the metric function $e^{-\Gamma}$ which has unacceptable incoming limits. The $M-$ shift technique changes the Weyl scalars while keeps the em energy momentum of the CEM problem unchanged. Under the light of all these considerations we present examples of scalar field solutions to some important CEM solutions.

\section{BELL - SZEKERES SOLUTION COUPLED WITH SCALAR FIELDS.}

CEM waves with constant profiles is known as the BS solution given by the line element

$$
d s^{2}=2 d u d v-\cos ^{2}(a u+b v) d x^{2}-\cos ^{2}(a u-b v) d y^{2}
$$

where $(a, b)$ are the constants of em fields. In the new coordinates

$$
\begin{gathered}
\tau=\sin (a u+b v) \\
\sigma=\sin (a u-b v)
\end{gathered}
$$

this line element takes the form

$$
d s^{2}=\frac{1}{2 a b}\left(\frac{d \tau^{2}}{\Delta}-\frac{d \sigma^{2}}{\delta}\right)-(\Delta d x)^{2}-(\delta d y)^{2}
$$

We note that these $(\tau, \sigma)$ coordinates can be obtained from the ones of previous section by letting $u \longrightarrow \sin (a u)$ and $v \longrightarrow \sin (b v)$. In the BS metric we have the case that $M=0$, therefore by the $M-$ shift we obtain

$$
d s^{2}=\frac{e^{-\Gamma}}{2 a b}\left(\frac{d \tau^{2}}{\Delta}-\frac{d \sigma^{2}}{\delta}\right)-(\Delta d x)^{2}-(\delta d y)^{2}
$$

Now by choosing the scalar field

$$
\phi(\tau, \sigma)=\alpha \tau \sigma+\frac{1}{4} \beta\left(3 \tau^{2}-1\right)\left(3 \sigma^{2}-1\right)
$$

as a solution of $(21)$, with $(\alpha, \beta)$ arbitrary constants, it enables us to integrate $\Gamma$ from (22) with the result

$$
\begin{aligned}
\Gamma= & \alpha^{2} \tau^{2}+\frac{9}{4} \beta^{2} \tau^{2}\left(1-\frac{\tau^{2}}{2}\right)-6 \alpha \beta \tau \sigma \Delta \delta+ \\
& \frac{\Delta}{4}\left\{\frac{9}{2} \beta^{2} \sigma^{2}\left(9 \tau^{2}-1\right)+\sigma^{2}\left(4 \alpha^{2}+9 \beta^{2}-45 \beta^{2} \tau^{2}\right)\right\}
\end{aligned}
$$


This function is well defined and finite as the $\mathrm{CH}$ at $\tau=1$ is approached. For $\tau \longrightarrow 1$, we have

$$
\Gamma(\tau \longrightarrow 1)=\alpha^{2}+\frac{9}{8} \beta^{2}
$$

This constitutes a non-singular extension of the BS solution in the presence of a scalar field. We consider the simpler case with $\beta=0$, which has the following scale invariant Weyl scalars

$$
\begin{aligned}
& \Psi_{2}^{(0)}=-\alpha^{2} a b \theta(u) \theta(v) \sin (2 a u) \sin (2 b v) \\
& \Psi_{4}^{(0)}=a^{2} \theta(v)\left[\delta(a u) \tan (b v)+\alpha^{2} \theta(u) \sin (2 a u) \sin (2 b v)\right] \\
& \Psi_{0}^{(0)}=b^{2} \theta(u)\left[\delta(b v) \tan (a u)+\alpha^{2} \theta(v) \sin (2 a u) \sin (2 b v)\right]
\end{aligned}
$$

The Ricci components are also given (in the Newman - Penrose formalism) by

$$
\begin{aligned}
& \Phi_{02}^{(0)}=-a b \theta(u) \theta(v) \\
& \Phi_{22}^{(0)}=a^{2} \theta(u)\left[1+\alpha^{2} \sin ^{2}(2 a u)\right] \\
& \Phi_{00}^{(0)}=b^{2} \theta(v)\left[1+\alpha^{2} \sin ^{2}(2 b v)\right] \\
& \Phi_{11}^{(0)}=-3 \Lambda^{(0)}=-\frac{1}{2} \alpha^{2} a b \theta(u) \theta(v) \sin (2 a u) \sin (2 b v)
\end{aligned}
$$

It is seen that with the exception of the distributional singularities on $(u=0, b v=\pi / 2)$ and $(v=0, a u=\pi / 2)$ to the future of the collision the metric is free of singularities.

We note that this scalar field extension of the BS metric also applies to its cross-polarized version easily. Since this is an exact back-reaction solution to the CEMS fields it provides an example that scalar field perturbations need not transform the $\mathrm{CH}$ into singularity. Different scalar fields, however, may not preserve the regularity of the $\mathrm{CH}$. Hence, it should not be wrong to conclude that, the stability or instability of CHs against scalar field perturbations depends crucially on the perturbing scalar field potential.

\section{SCALAR FIELD EXTENSION OF THE HBB SOLUTION.}

An interesting solution in the CEM waves was given by HBB which represents collision of an impulsive gravitational wave with a wave packet consisting of superposed impulsive gravitational wave and a shock em wave. This solution naturally possesses both the Khan Penrose [15] and Griffiths [16] limits and does not belong to any known family of solutions [1]. In this section we show that by the $M-$ shift we can add a scalar field to the colliding fields to extend them into more complex wave packets.

The incoming metrics in the HBB problem are [12] 


$$
\begin{gathered}
d s^{2}=2 d u d v-(1+k u)^{2} d x^{2}-(1-k u)^{2} d y^{2},(\text { Region II }) \\
d s^{2}=2 d u d v-\left(\cos b v+\frac{l}{b} \sin b v\right)^{2} d x^{2}-\left(\cos b v-\frac{l}{b} \sin b v\right)^{2} d y^{2},(\text { Region III) }
\end{gathered}
$$

in which the null coordinates are to be multiplied with the step functions. Here, $k$ and $l$ are the impulsive gravitational constants while $b$ represents the em constant. We note that our coordinate $v$ (Region III) is different from the one employed by HBB, i.e. the relation is

$$
v \longrightarrow \frac{1}{b} \tan (b v)
$$

so that in the limit, $b \longrightarrow 0$ they coincide. The metric functions and the em field strengths found by HBB are

$$
\begin{aligned}
e^{-U} & =F \cos ^{2} b v \\
e^{V} & =\frac{1+k u B+A \sqrt{1-B^{2}}}{1-k u B-A \sqrt{1-B^{2}}} \\
e^{-M} & =\frac{H^{2}}{A B \sqrt{F}} \\
\phi_{2} & =\frac{-k B \tan b v}{A H \sqrt{F}} \\
\phi_{0} & =\frac{b\left[k u\left(\frac{l^{2}+b^{2}}{l^{2}}\right)\left(1-B^{2}\right)^{3 / 2}+A B^{3}\right]}{B H \sqrt{F}}
\end{aligned}
$$

where the notation is

$$
\begin{aligned}
& F=A^{2}+B^{2}-1-k^{2} u^{2} \tan ^{2} b v \\
& H=A B-k u \sqrt{1-B^{2}}
\end{aligned}
$$

and

$$
\begin{aligned}
& A=\sqrt{1-k^{2} u^{2}} \\
& B=\sqrt{1-\frac{l^{2}}{b^{2}} \tan ^{2} b v}
\end{aligned}
$$

Our new coordinates appropriate for the present problem are

$$
\begin{aligned}
\tau & =B \cos b v \sqrt{1-A^{2}}+A \sqrt{1-B^{2} \cos ^{2} b v} \\
\sigma & =B \cos b v \sqrt{1-A^{2}}-A \sqrt{1-B^{2} \cos ^{2} b v}
\end{aligned}
$$

so that the metric function $U$ is expressed by 


$$
e^{-U}=\sqrt{1-\tau^{2}} \sqrt{1-\sigma^{2}}
$$

Solution of the scalar field equation (21) in the present coordinates can easily be found. We present two particular solutions.

a) Let

$$
\phi(\tau, \sigma)=\alpha \tau \sigma
$$

where $\alpha=$ constant, and integration of $\Gamma$ function from $\mathrm{Eq}(22)$ results in

$$
\Gamma=\alpha^{2}\left(\tau^{2}+\sigma^{2}-\tau^{2} \sigma^{2}\right)
$$

This choice of scalar field occurs from both sides of the incoming waves and it is regular. The em field strengths remain unchanged.

b) Let

$$
\phi(\tau, \sigma)=\left\{\begin{array}{c}
=\beta \tanh ^{-1}\left(\frac{\tau+\sigma}{1+\tau \sigma}\right) \quad(\text { Region IV }, \quad u>0, \quad v>0) \\
=0, \quad(\text { Region III, } \quad u \leq 0)
\end{array}\right.
$$

where $\beta=$ constant. The $\Gamma$ function now becomes

$$
e^{-\Gamma}=\left[\frac{\left(1-\tau^{2}\right)\left(1-\sigma^{2}\right)}{(\tau+\sigma)^{4}}\right]^{\beta^{2}}
$$

In this particular class the scalar field exists only for $u>0$, which in the Region II $(\mathrm{v}<0)$ takes the form

$$
e^{-\Gamma}=\left(\frac{1-k^{2} u^{2}}{4 k^{2} u^{2}}\right)^{2 \beta^{2}}
$$

and is well-defined. This solitonic scalar field occurs only in Region II and IV while in Region III there is no scalar field. Hence, Region II contains gravity + scalar waves while Region III contains gravity +em waves.

\section{CEMS WAVES ISOMETRIC TO THE PENNEY SOLUTION.}

As another example we consider a solution for CEMS waves which is transformable to the spherically symmetric geometry. Unlike the two previous examples the present one has not been obtained by the $M-$ shift method. In spherically symmetric problem by the uniqueness arguments Reissner - Nordström solution is the single available black hole solution. Scalar field extension of this metric was found long ago by Penney [13]. The result was that inclusion of scalar field converted both horizons into spacetime singularities which naturally destroyed the black hole property. By the same token solution in CEMS waves that is isometric to spherically symmetric geometry no different result other than a metric plagued with singularities is expected.

The metric, scalar field and the em vector potential in the Region II are given respectively as follows 


$$
\begin{aligned}
d s^{2} & =Z^{2}\left(1-u^{2} \theta(u)\right)^{\frac{1}{2}-A}\left(4 d u d v-\left(1-u^{2} \theta(u)\right)^{\frac{3}{2}} d x^{2}\right)-Z^{-2}\left(1-u^{2} \theta(u)\right)^{A} d y^{2} \\
\phi(u) & =\frac{1}{2} \sqrt{1-A^{2}} \ln \left|\frac{1+u \theta(u)}{1-u \theta(u)}\right| \\
A_{\mu}(u) & =2 \sqrt{|a b|} \delta_{\mu}^{x} A u \theta(u)
\end{aligned}
$$

where $2 Z(u)=a(1+u \theta(u))^{A}+b(1-u \theta(u))^{A},(a, b)$ are the constant em parameters, and $0 \leq A \leq 1$ is the constant scalar field parameter. Unfortunately this data has diverging energy- momentum $T_{u u}$ and Weyl scalar $\Psi_{4}$ at $u=1$. Replacing $u \longleftrightarrow v\left(-v\right.$ in $\left.A_{\mu}\right)$ specifies also the initial data in the incoming Region III. The solution of these CEMS waves is

$$
\begin{aligned}
d s^{2} & =\Delta^{1-A} Z^{2}\left(\frac{d \tau^{2}}{\Delta}-\frac{d \sigma^{2}}{\delta}-\delta d x^{2}\right)-\Delta^{A} Z^{-2} d y^{2} \\
\phi(\tau) & =\frac{1}{2} \sqrt{1-A^{2}} \ln \left|\frac{1+\tau}{1-\tau}\right| \\
A_{\mu} & =2 \delta_{\mu}^{x} \sqrt{|a b|} A \sigma
\end{aligned}
$$

where $(\tau, \sigma)$ coordinates are as in $(18)$ and

$$
2 Z=a(1+\tau)^{A}+b(1-\tau)^{A}
$$

We note that this solution is invariant under $A \longrightarrow-A$ therefore it is sufficient to consider the case $0 \leq A \leq 1$. As particular limits of (47) we observe the following cases.

i) For $A=1$ (and $a=b$ ), it reduces to the well known BS solution of CEM waves which is regular. This admits a $\mathrm{CH}$ at $\tau=1(0<\sigma<1)$ and null singular points at $\tau=1, \sigma= \pm 1$ (i.e. $u=1, v=0$ and $v=1, u=0$ ).

ii) For $A=0$, it reduces to colliding Einstein - Scalar waves with a spacelike singularity at $\tau=1$. Let us note that, it is still an open problem to find colliding pure scalar waves without singularities.

iii) For $0<A<1$ we have an example of CEMS waves solution with a spacelike singularity at $\tau=1$. Further, at $\tau=1$ the metric becomes completely degenerate, i.e. $d s^{2}=0$.

In order to see the role of the scalar field in directing the geodesics of a particle in the interaction region we find the proper time of fall into the singularity.

The proper time of fall into the singularity is given by

$$
t_{0}=\int_{0}^{1} \frac{Z^{2}}{\sqrt{\delta_{1} \Delta^{A} Z^{2}+\alpha^{2} \Delta^{2 A-1}}} d \tau
$$

where $\alpha$ is a constant associated with a cyclic coordinate and $\delta_{1}=0$ (for null) or $\delta_{1}=1$ (for timelike) geodesics. We obtain 


$$
t_{0}=\left\{\begin{array}{c}
\frac{a^{2}}{\alpha} B_{\frac{1}{2}}\left[\frac{3}{2}-A, \frac{3}{2}+A\right]+\frac{b^{2}}{\alpha} B_{\frac{1}{2}}\left[\frac{3}{2}+A, \frac{3}{2}-A\right]+\frac{a b \pi}{8 \alpha},(\text { null }- \text { geodesics }) \\
a B_{\frac{1}{2}}\left[1-\frac{A}{2}, 1+\frac{A}{2}\right]+b B_{\frac{1}{2}}\left[1+\frac{A}{2}, 1-\frac{A}{2}\right],(\text { timelike }- \text { geodesics })
\end{array}\right.
$$

in which $B_{\lambda}(\mu, \nu)$ is an incomplete beta function defined by

$$
\begin{aligned}
B_{\lambda}[\mu, \nu]= & \int_{0}^{\lambda} t^{\mu-1}(1-t)^{\nu-1} d t=\mu^{-1} \lambda^{\mu} F(\mu, 1-\nu ; \mu+1 ; \lambda) \\
& 0 \leq \lambda \leq 1 \\
& \mu, \nu>0
\end{aligned}
$$

Finally we prove the local equivalence of our metric with that of Penney [13]. By choosing

$$
2 Z=a_{0}|1+\tau|^{A}-b_{0}|1-\tau|^{A}
$$

and using the transformation

$$
\tau=\frac{m-r}{\sqrt{m^{2}-Q^{2}}}, \quad x=\phi, \quad y=\left(\sqrt{m^{2}-Q^{2}}\right) t, \quad \sigma=\cos \theta
$$

with $Q^{2}=\frac{e^{2}}{A^{2}}$, where $e$ is an electric charge, transforms our metric (47) into

$$
d s^{2}=e^{-\alpha} d t^{2}-e^{\alpha} d r^{2}-e^{\beta}\left(d \theta^{2}+\sin ^{2} \theta d \phi^{2}\right)
$$

Here we have

$$
\begin{gathered}
e^{\alpha}=\left[\left(r-a_{0}\right)\left(r-b_{0}\right)\right]^{-A}\left\{\frac{b_{0}\left|r-a_{0}\right|^{A}-a_{0}\left|r-b_{0}\right|^{A}}{b_{0}-a_{0}}\right\}^{2} \\
e^{\beta}=\left[\left(r-a_{0}\right)\left(r-b_{0}\right)\right] e^{\alpha} \\
a_{0}=m-\sqrt{m^{2}-\frac{e^{2}}{A^{2}}} \\
b_{0}=m+\sqrt{m^{2}-\frac{e^{2}}{A^{2}}}
\end{gathered}
$$

Metric (53) is recognized as the solution of Penney, representing a singular scalar field extension of the Reissner - Nordstrom geometry.

\section{DISCUSSION.}

We presented a method that adds scalar fields to any known EM solution in CPWs. Physically interesting case is to find solutions without singularities. This seems possible when the background CEM metric is singularity free in the interaction region. Any solution that is already singular becomes worse with the addition of scalar fields. So far no singularity free colliding pure scalar field solution has been found. In the solution in section IV, we see dramatically how the addition of the scalar field parameter $0<A<1$ makes spacetime singular. The $M$ - shift technique applies equally well to any vacuum metric. The resulting superposition of plane waves with scalar fields is equivalent to the collision of wavepackets

Acknowledgement 2 . We wish to thank Dr. Andrew Shoom for fruitful discussions. 


\section{REFERENCES}

[1] Griffiths, J.B. (1991). Colliding Plane Waves in General Relativity: Oxford University Press, Oxford.

[2] Chandrasekhar, S. and Xanthopoulos, B.C. (1986). Proc. R. Soc. London A $408,175$.

[3] Yurtsever, U. (1987). Phys. Rev. D 36, 1662.

[4] Bell, P. and Szekeres, P. (1974). Gen. Rel. Grav. 5, 275.

[5] Chandrasekhar, S. and Xanthopoulos, B.C. (1985) Proc. R. Soc. London A $398,223$.

[6] Halilsoy, M. (1990). J. Math. Phys. 31, 2694.

[7] Chao, W.Z. (1982). J. Phys. A: Math. Gen. 15, 2429.

[8] Halilsoy, M. (1985). Lett. Nuovo Cimento, B 44, 544.

[9] Choptuik, M.W. (1993). Phys. Rev. Lett. 70, 9.

[10] Burko, L.M. (1997). Phys. Rev. Lett. 79, 4958.

[11] Eardley, D.M. and Giddings, S.B. (2002). Phys. Rev. D66, 044011.

[12] Hogan, P.A., Barrabes, C. and Bressange, G.F. (1998) Lett. Math. Phys. 43, 263.

[13] Penney, R. (1969). Phys. Rev. 182,1383.

[14] Eris, A. and Gurses, M. (1977). J. Math.Phys. 16, No.7, 1303.

[15] Khan, K.A. and Penrose, R. (1971). Nature (London). 229, 185.

[16] Griffiths, J.B. (1975). Phys. Lett. A, 54, 269. 